\title{
The neuropathology of schizophrenia: new insights from postmortem studies
}

\author{
Andrea Schmitt $\cdot$ Peter Falkai
}

Published online: 6 May 2014

(c) Springer-Verlag Berlin Heidelberg 2014

Brain imaging studies contributed substantially to advanced insights into alterations of specific brain circuits in schizophrenia. For example, in a functional magnetic resonance imaging (fMRI) study, Bergé et al. [1] determined brain activation during facial emotion discrimination in first-episode schizophrenia patients before starting antipsychotic treatment and after clinical improvement. At baseline, in ventro-limbic regions, including hippocampus and amygdalae, ventral-posterior regions and fronto-temporal regions, hypoactivation could be detected. In contrast, after treatment and clinical improvement, activity increased in lingual gyrus, calcarine fissure and amygdalae during anger discrimination. The neuropathology underlying decreased activation or volumetric deficits on the cellular and molecular level is, however, unknown, and postmortem investigations may contribute to provide pathophysiological insights.

According to the dopamine hypothesis, antipsychotics target dopaminergic neurons, and a dopaminergic dysfunction may be the basis of psychotic-positive symptoms. In a postmortem study, Williams et al. [2] examined the cytoarchitecture of dopaminergic neurons in the substantia nigra in patients with schizophrenia, depression and healthy controls. In schizophrenia patients, but not those with depression, increased nucleolar volume, cross-sectional area and length of dopaminergic neurons were observed, probably reflecting alterations in neuronal function and gene expression. In a genome-wide microarray study, Sellmann et al. [3] compared gene expression in the left

A. Schmitt $(\bowtie) \cdot$ P. Falkai

Department of Psychiatry and Psychotherapy,

Ludwig-Maximilians-University Munich, Nußbaumstr. 7,

80336 Munich, Germany

e-mail: andrea.schmitt@med.uni-muenchen.de superior temporal cortex between chronic schizophrenia patients and healthy controls. The authors applied statistical tests and dimensionality reduction methods to characterize differentially expressed genes. The set of upregulated genes included those involved in gene transcription, histone methylation as an epigenetic marker, apoptosis and hypoxic events. In postmortem tissue, however, several cell types such as neurons and glial cells contribute to the findings. In the corpus callosum, composed of white matter and repeatedly shown as decreased in schizophrenia, Steiner et al. [4] report downregulation of S100B in the nuclear proteome using mass spectrometry and Western blotting. S100B is expressed and involved in maturation of oligodendrocytes, which have been shown to be decreased in fronto-hippocampal brain regions. Therefore, a dysfunction of oligodendroglia as basis of a myelination deficit comes closer into the focus of the schizophrenia pathophysiology. Moreover, an atypical degenerative component has been discussed to contribute to disease progression. Investigating late-onset schizophrenia, affective disorders and healthy controls, Nagao et al. [5] found higher frequency of Lewy body disease, argyrophilic grain disease and corticobasal degeneration in late-onset schizophrenia compared to healthy controls, but not in affective disorders generally. Interestingly, argyrophilic grains were restricted to the limbic system and temporal cortex, which is consistent with the finding that delusion was significantly more frequent in patients with argyrophilic grain disease.

Apart from positive psychotic symptoms, the described neuropathological alterations may contribute to cognitive deficits. Gilbert et al. [6] used alterations in cognitive domains to stratify schizophrenia patients with respect to outcome measures. Patients with selective cognitive impairments demonstrated better improvement at outcome, whereas generally impaired patients were more likely to be 
treatment refractory. In a candidate gene study, Lencer et al. [7] investigated first-episode schizophrenia patients genotyped for eight markers in DRD2 and GRM3 encoding for the metabotropic glutamate receptor 3 with respect to eye movement deficits at baseline and after antipsychotic treatment. They found relationships for 4 markers and thus demonstrated measures of motor and neurocognitive deficits to be associated with genes regulating the dopamine and glutamate system.

Finally, Williams et al. [8] report a decrease in astrocytes in the white matter of subgenual cingulate gyrus in schizophrenia patients. In the substantia nigra, they also show a decrease in astrocyte density [2]. This is interesting because an astrocyte dysfunction may lead to neurotrophic deficits and impaired astrocyte-neuron interaction in these brain regions. Consistent with these results, no astrocytosis has been found in schizophrenia, suggesting that schizophrenia is not a classical neurodegenerative disease, but rather entails disturbances of synaptic connectivity. Future studies should investigate synaptic plasticity and oligodendrocyte pathology in more detail. Gaining insight into these pathophysiological processes may extend our understanding of altered molecular pathways and may lead to the development of new treatment strategies for schizophrenia.

\section{References}

1. Bergé D, Carmona S, Salgado P, Rovira M, Bulbena A, Vilarroya O (2013) Limbic activity in antipsychotic naïve first-episode psychotic subjects during facial emotion discrimination. Eur Arch Psychiatry Clin Neurosci. doi:10.1007/s00406-013-0465-5

2. Williams MR, Galvin K, O'Sullivan B, Macdonald CD, Ching EW, Turkheimer F, Howes OD, Pearce RK, Hirsch SR, Maier M (2013) Neuropathological changes in the substantia nigra in schizophrenia but not depression. Eur Arch Psychiatry Clin Neurosci. doi:10.1007/s00406-013-0479-z

3. Sellmann C, Pildaín LV, Schmitt A, Leonardi-Essmann F, Durrenberger PF, Spanagel R, Arzberger T, Kretzschmar H, Zink M, Gruber O, Herrera-Marschitz M, Reynolds R, Falkai P, Gebicke-Haerter PJ, Matthäus F (2013) Gene expression in superior temporal cortex of schizophrenia patients. Eur Arch Psychiatry Clin Neurosci. doi:10.1007/s00406-013-0473-5

4. Steiner J, Schmitt A, Schroeter ML, Bogerts B, Falkai P, Turck CW, Martins-de-Souza D (2014) S100B is downregulated in the nuclear proteome of schizophrenia corpus callosum. Eur Arch Psychiatry Clin Neurosci. doi:10.1007/s00406-014-0490-z

5. Nagao S, Yokota O, Ikeda C, Takeda N, Ishizu H, Kuroda S, Sudo K, Terada S, Murayama S, Uchitomi Y (2013) Argyrophilic grain disease as a neurodegenerative substrate in late-onset schizophrenia and delusional disorders. Eur Arch Psychiatry Clin Neurosci. doi:10.1007/s00406-013-0472-6

6. Gilbert E, Mérette C, Jomphe V, Emond C, Rouleau N, Bouchard RH, Roy MA, Paccalet T, Maziade M (2013) Cluster analysis of cognitive deficits may mark heterogeneity in schizophrenia in terms of outcome and response to treatment. Eur Arch Psychiatry Clin Neurosci. doi:10.1007/s00406-013-0463-7

7. Lencer R, Bishop JR, Harris MS, Reilly JL, Patel S, Kittles R, Prasad KM, Nimgaonkar VL, Keshavan MS, Sweeney JA (2013) Association of variants in DRD2 and GRM3 with motor and cognitive function in first-episode psychosis. Eur Arch Psychiatry Clin Neurosci. doi:10.1007/s00406-013-0464-6

8. Williams M, Pearce RK, Hirsch SR, Ansorge O, Thom M, Maier M (2013) Fibrillary astrocytes are decreased in the subgenual cingulate in schizophrenia. Eur Arch Psychiatry Clin Neurosci. doi:10.1007/s00406-013-0482-4 